\title{
Procédés de fabrication et propriétés physiques des couvertes de poteries romaines : une approche physico-chimique multi-échelle
}

\section{Le succès exceptionnel}

de la sigillée, vaisselle de table

en terre cuite de la période

romaine, est en grande partie

dû à la brillance et à la couleur

rouge uniforme de sa couverte

qui ont nécessité

le développement de procédés

techniques très performants.

Dans cet article nous comparons

les réalisations italiennes

aux productions gauloises qui

leur ont succédé, sous le regard

de la science des matériaux.

Nous montrons que l'étude des

couvertes, en plus de permettre

d'en comprendre la fabrication,

a mis en évidence une évolution

majeure de leurs propriétés

physiques que l'on peut relier

à une évolution de leur utilisation.

Les termes en gras suivis d'un astérisque sont définis dans le glossaire, p. 42.

Philippe Sciau (philippe.sciau@cemes.fr) et Jesse Groenen

CEMES (CNRS et Université de Toulouse), 29 rue J. Marvig, 31055 Toulouse

La céramique sigillée [1], ou Terra sigillata $^{\star}$, est une vaisselle de table emblématique de la période romaine. Fabriquée en grande quantité et de façon standardisée dans un nombre restreint d'ateliers, elle était distribuée sur tout le territoire romain.

Les premières formes de sigillée avec des motifs en relief datent du $1^{\text {er }}$ siècle avant J.-C., mais ce n'est que quelques décennies plus tard que se développe véritablement la sigillée de qualité avec la mise au point de cuissons en conditions oxydantes permettant l'obtention de couvertes $^{\star}$ brillantes d'une couleur rouge uniforme. Les premières sigillées de qualité furent produites en Italie centrale à Arezzo vers le milieu du $1^{\text {er }}$ siècle avant J.-C. et leur diffusion s'étend dès les années 40-30 avant J.-C. Ce fut un vecteur important de la romanisation en diffusant, à travers ses décors et son utilisation comme vaisselle de table, la culture romaine. La diffusion de la sigillée s'intensifie fortement avec l'extension du monde romain à la fin de la République et au début de l'Empire, et conduit à la mise en place de nouveaux centres de production, notamment dans le sud de la Gaule.

La figure 1 donne un exemple de l'ampleur de la diffusion de la céramique élaborée dans l'un de ces centres de production, La Graufesenque, situé près de Millau (Aveyron) [2].

\section{La couverte d'une sigillée}

En dehors de l'esthétique de ses décors (fig. 2), la qualité d'une sigillée est directement liée à l'éclat et à la couleur de sa couverte ou " engobe ». Cette dernière, d'une épaisseur de quelques dizaines de micromètres, se forme durant la cuisson par vitrification sous atmosphère oxydante d'une préparation argileuse riche en fer. Cette préparation est élaborée par lessivage et décantation d'une argile non calcaire chargée en potassium et naturellement riche en oxydes et/ou hydroxydes de fer. Ce procédé permet d'éliminer les matières organiques et de ne conserver que la partie fine, riche en minéraux argileux et en composés de fer. Mise en suspension dans l'eau, cette préparation est ensuite apposée sur le vase cru au pinceau ou, dans le cas des sigillées, par trempage du vase comme en attestent les marques de doigts observées sur les pieds de vases antiques. La plus faible granulométrie et la plus forte teneur en éléments alcalins ( $\mathrm{Na}, \mathrm{K}$, qui abaissent la température de vitrification) de l'engobe permettent de le vitrifier sans vitrifier le corps du vase et ainsi sans induire une déformation de ce dernier. Toutefois, comme le corps des sigillées, appelé aussi la "pâte ", était également façonné à partir d'une préparation à grains fins, riche en potassium (tableau 1), la différence de température de vitrification est faible et l'obtention de sigillées présentant une 


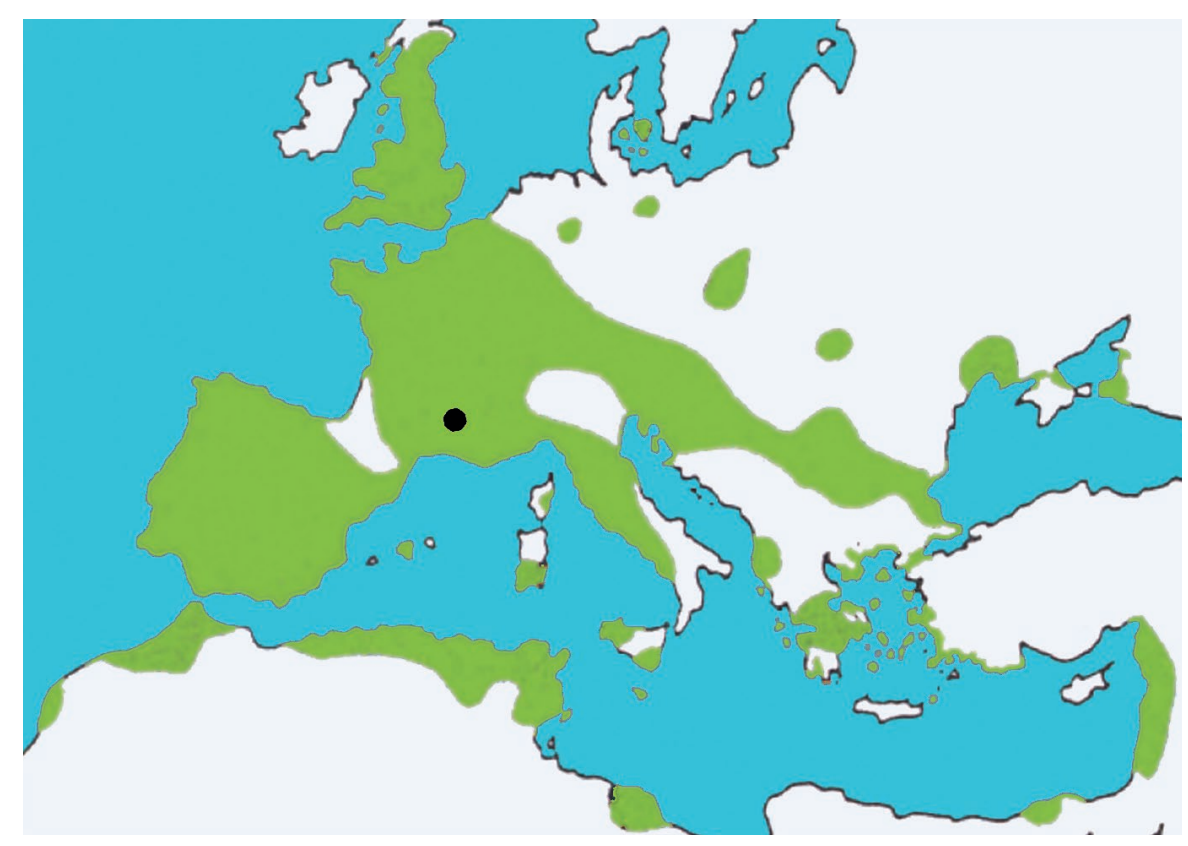

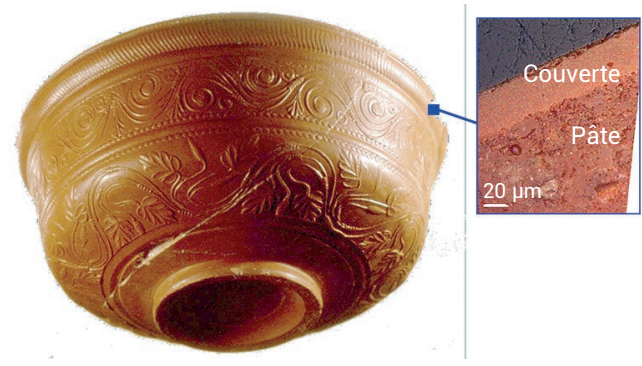

2. Vase décoré de la Graufesenque (photo Alain Vernhet) et observation en microscopie optique d'une coupe polie transverse montrant la couverte et le corps du vase. On peut aussi utiliser le terme de « pâte » pour caractériser ce dernier.

1. Carte de diffusion des céramiques produites dans le centre de La Graufesenque (O) situé à côté de Millau (Aveyron).

couverte bien brillante demande une bonne maitrise du processus de cuisson.

La vitrification doit également être réalisée sous atmosphère oxydante, afin d'obtenir une couleur rouge uniforme et d'empêcher la formation de composés à base de fer bivalent $\left(\mathrm{Fe}^{2+}\right)$ tels que la magnétite $\left(\mathrm{Fe}^{2+} \mathrm{Fe}_{2}{ }^{3+} \mathrm{O}_{4}\right)$ ou l'hercynite $\left(\mathrm{Fe}^{2+} \mathrm{Al}_{2}{ }^{3+} \mathrm{O}_{4}\right)$, dont la présence entraine un brunissement, voire un noircissement de la couleur de la couverte. Pour maintenir une atmosphère oxydante autour des vases, les potiers romains ont cherché à les isoler des gaz de combustion et des fumées soit en les enfermant dans des casettes $^{\star}$, soit en canalisant les gaz et fumées au moyen de tubes en terre cuite ou « tubulures». De nombreuses tubulures ont été retrouvées sur les lieux de production, notamment ceux datés des $1^{\text {er }}$ et $2^{\mathrm{e}}$ siècles après J.-C., mais jusqu'à présent aucun four suffisamment bien conservé n'a pu être mis au jour pour pouvoir en reconstituer la structure et le fonctionnement. Des reproductions modernes de fours à tubulures ont toutefois montré qu'ils étaient particulièrement bien adaptés à la cuisson de sigillées et permettaient, grâce à un chauffage par rayonnement, d'obtenir de belles couvertes rouges brillantes.

\section{D'un point de vue science des matériaux}

Les couvertes de sigillées sont constituées d'une phase vitreuse, représentant entre 60 et $80 \%$ de la masse totale, et de divers cristaux d'oxydes dont les tailles varient de quelques dizaines de nanomètres à quelques micromètres [3]. Certains de ces cristaux, notamment les plus gros, proviennent de la fraction sableuse conservée lors de la préparation

\begin{tabular}{|c|c|c|c|c|c|c|c|c|}
\hline & & $\mathrm{SiO}_{2}$ & $\mathrm{Al}_{2} \mathrm{O}_{3}$ & $\mathrm{Fe}_{2} \mathrm{O}_{3}$ & $\mathrm{~K}_{2} \mathrm{O}$ & $\mathrm{MgO}$ & $\mathrm{CaO}$ & $\mathrm{TiO}_{2}$ \\
\hline \multirow{2}{*}{$\begin{array}{l}\text { Italiennes } \\
\text { (Arezzo) }\end{array}$} & Couvertes & 49,5 & 26,9 & 10,5 & 6,6 & 3,2 & 1,5 & 0,6 \\
\hline & Pâtes & 56,9 & 18,4 & 7,8 & 2,6 & 3,0 & 10,4 & 0,9 \\
\hline \multirow{2}{*}{$\begin{array}{c}\text { Gauloises } \\
\text { (La Graufesenque) }\end{array}$} & Couvertes & 55,9 & 23,1 & 9,6 & 8,1 & 1,0 & 1,3 & 0,7 \\
\hline & Pâtes & 52,3 & 22,6 & 6,3 & 3,8 & 2,0 & 11,9 & 1,1 \\
\hline
\end{tabular}

Tableau 1. Compositions chimiques (pourcentages) des couvertes et pâtes, données en équivalent de masse d'oxyde. 
$>>$

et sont essentiellement des grains de quartz. Les autres sont formés lors de la cuisson par réactions chimiques, à partir principalement de la transformation et recristallisation des oxydes et hydroxydes de fer et de la déstructuration des minerais argileux. La couverte d'une sigillée est un milieu complexe dont l'étude physico-chimique permet d'obtenir des informations sur les différentes phases de fabrication (nature de l'argile utilisée, préparation de l'engobe et cuisson), mais également sur les propriétés physiques. Pour cela, il faut toutefois prendre en compte l'hétérogénéité de ces matériaux, ainsi que la variabilité de ce type de production. C'est ce que nous allons essayer de montrer à travers une étude comparative de deux grandes catégories de sigillées : celles produites en Italie centrale (Arezzo) à la fin de la deuxième moitié du $1^{\mathrm{er}}$ siècle avant J.-C., et celles produites au $1^{\text {er }}$ siècle après J.-C. dans le grand centre de La Graufesenque, au sud de la Gaule.

\section{Les analyses physico-chimiques à l'échelle microscopique}

S'il est relativement facile pour un céramologue de la période romaine de séparer les productions de ces deux zones d'après leur style et leur aspect, il n'en reste pas moins vrai que la couleur et la brillance de leurs couvertes sont très comparables. En revanche, la nature des défauts (fig. 3a) est toujours différente [3]. L'adhérence des couvertes italiennes est plus faible et, sur de nombreux fragments, on observe des zones écaillées. Les couvertes gauloises, quant à elles, présentent très souvent des fissures. De simples observations de coupes transverses en microscopie électronique à balayage (MEB) permettent de comprendre l'origine de ces défauts (fig. 3b). Les couvertes italiennes sont très bien vitrifiées, mais pas les pâtes. Il en résulte une zone de fragilité à l'interface couverte/pâte bien visible sur les images MEB. A contrario, la pâte des sigillées gauloises est partiellement vitrifiée. L'interface ne correspond pas à une zone de fragilité, mais les contraintes dues aux différences de coefficient de dilatation entre la pâte et la couverte peuvent entrainer, lors du refroidissement, l'apparition de fissures dans la couverte si ce refroidissement n'est pas suffisamment

a
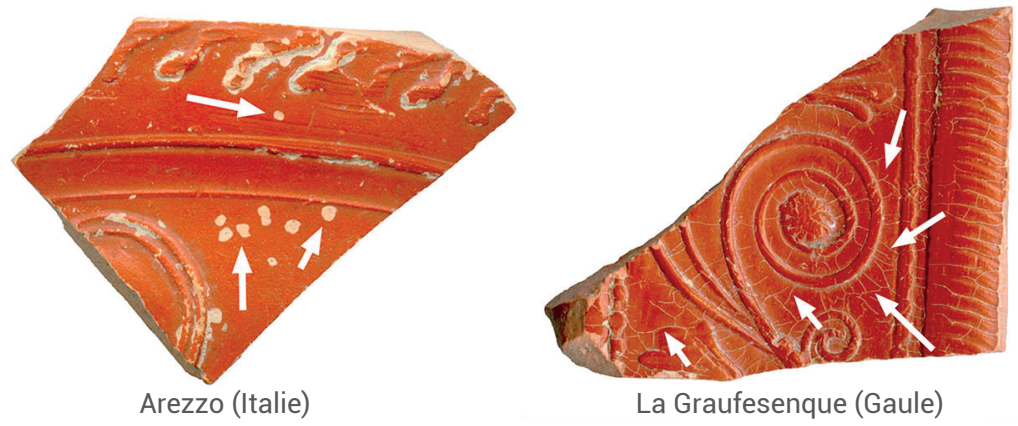

b
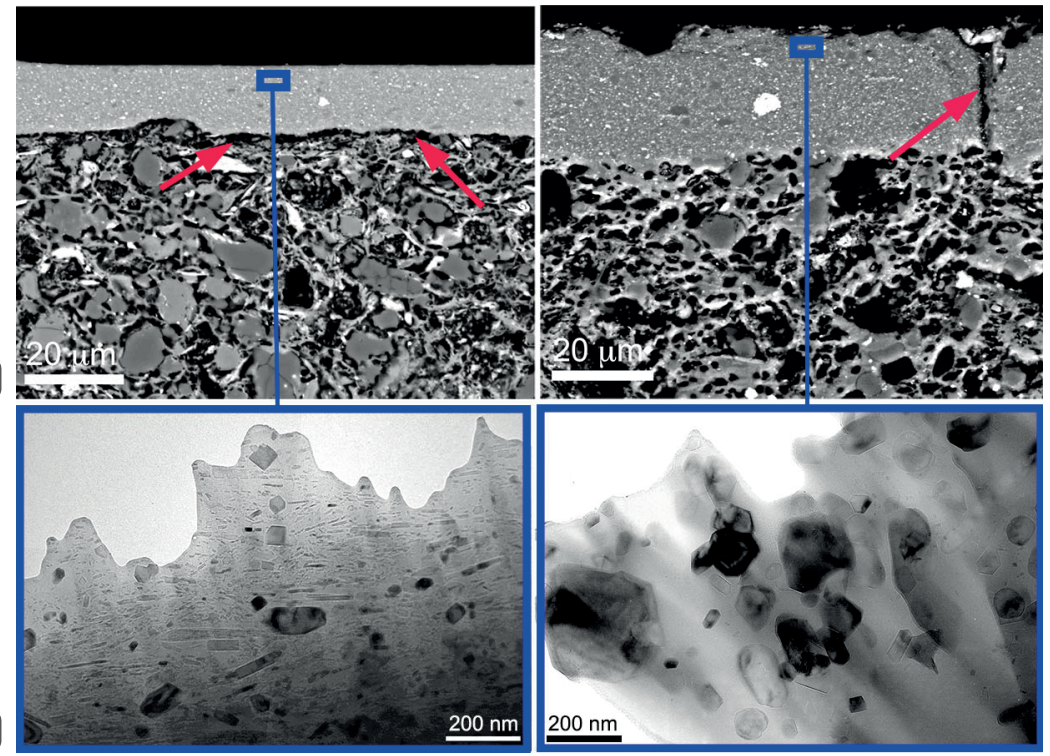

3. Étude comparative de sigillées d'Arezzo et de la Graufesenque [3].

(a) Photographies des défauts des couvertes (flèches blanches).

(b) Observations par MEB des coupes transverses montrant les différences de texture et les zones de fragilité (flèches rouges).

(c) Observations des structures des couvertes (zones entourées de bleu dans les figures $3 \mathrm{~b}$ ) par microscopie électronique en transmission.

lent. Les couvertes gauloises sont plus hétérogènes, avec des grains de quartz plus nombreux et plus gros. La décantation a été moins poussée et une part plus importante de la partie sableuse a été conservée. Cela pourrait résulter soit d'une préparation plus rapide et moins soigneuse, soit d'une volonté délibérée de réduire la fissuration. De fait, la présence de grains de quartz micrométriques diminue le coefficient de dilatation de la couverte et atténue les contraintes lors du refroidissement.

Les analyses des compositions chimiques et minérales ont également mis en évidence d'intéressantes différences entre les deux groupes (tableau 1, p. 39). Dans les deux cas, les pâtes ont été obtenues à partir d'argiles calcaires et les couvertes à partir d'argiles non calcaires riches en fer et en potassium. On remarquera toutefois que les couvertes gauloises ont des taux de magnésium $(\mathrm{MgO} \leq 1 \%$ en masse) bien inférieurs à leurs homologues italiennes. Cette différence se retrouve dans la composition minérale identifiée par diffraction des rayons X. Les phases cristallines principales des couvertes gauloises sont le quartz $\left(\mathrm{SiO}_{2}\right)$, l'hématite $\left(\mathrm{Fe}_{2} \mathrm{O}_{3}\right)$ et le corindon $\left(\mathrm{Al}_{2} \mathrm{O}_{3}\right)$, tandis que dans les couvertes italiennes on retrouve du quartz, de l'hématite et du spinelle $\left(\mathrm{MgAl}_{2} \mathrm{O}_{4}\right)$ en phases principales, mais aucun corindon. Le spinelle représente au moins 30\% de la masse de la partie cristalline des couvertes italiennes, alors qu'il n'est qu'en très faible proportion $(<2 \%)$ dans les couvertes gauloises

Des études du comportement en température de la fraction fine d'argiles illitiques naturelles de compositions proches de celles des couvertes a permis de comprendre l'origine de cette différence. Quand la teneur en $\mathrm{MgO}$ est supérieure 
à 2-2,5\% comme dans les couvertes italiennes, du spinelle commence à se former dès la fin de la décomposition de l'illite ${ }^{\star}$ vers $850^{\circ} \mathrm{C}$. Lors de cette décomposition, les groupements $\left(\mathrm{AlO}_{6}\right)$ de l'illite réagissent avec les ions $\mathrm{Mg}^{2+}$ pour former des cristaux de spinelle $\mathrm{MgAl}_{2} \mathrm{O}_{4}$, et le résidu riche en groupements $\mathrm{SiO}_{4}$ peut alors plus facilement participer à la formation de la phase vitreuse. En revanche, si le magnésium est en trop faible quantité comme dans les couvertes gauloises, la décomposition de l'illite donne naissance à un silicate d'aluminium potassique qui ne se transforme en phase vitreuse qu'au-dessus de $1000^{\circ} \mathrm{C}$; l'excès d'ions $\mathrm{Al}^{3+}$ de la phase vitreuse entraine la formation de cristaux nanométriques de corindon.

L'atmosphère oxydante favorise les ions ferriques $\left(\mathrm{Fe}^{3+}\right)$, et les différents oxydes et hydroxydes de fer se transforment en hématite. En revanche, si l'atmosphère est réductrice (présence de fumée et des gaz de combustion), c'est la forme ferreuse $\left(\mathrm{Fe}^{2+}\right)$ qui est favorisée et entraine un noircissement de la couverte.

\section{Les analyses physico-chimiques à l'échelle nanométrique}

À cette échelle, une couverte gauloise est constituée d'une matrice vitreuse dense contenant principalement des cristaux d'hématite de taille submicrométrique, et des cristaux de corindon, plus petits et aux formes plus arrondies (fig. $3 \mathrm{c}$ de droite). Les analyses locales ont mis en évidence la présence de titane et d'aluminium dans les cristaux d'hématite, et celle de fer dans ceux de corindon [3, 4].

Les couvertes italiennes sont aussi composées d'une matrice vitreuse dense et de cristaux d'hématite, mais les cristaux de corindon sont remplacés par des cristaux de spinelle de forme parallélépipédique fortement orientés (fig. 3c de gauche). Quand la préparation argileuse est apposée sur le vase, les cristaux d'illite, en forme de plaquettes, ont tendance à se poser sur la surface à la manière de feuilles de papier jetées sur une table. Lors de la cuisson, les cristaux de spinelle se forment à partir de l'illite et conservent en grande partie l'orientation de ces derniers. Les cristaux d'hématite de ces couvertes contiennent également du titane et de l'aluminium.
Ces substitutions entrainent des modifications des paramètres des mailles cristallines, qui ont été mesurés par diffraction des rayons X. Elles ont aussi une influence sur les modes de vibration, qui ont été étudiés par spectroscopie Raman. C'est en particulier le cas de l'hématite, où les substitutions du fer par l'aluminium et le titane modifient les fréquences de vibration des modes Raman et font apparaitre, en raison de la brisure de symétrie qu'elles provoquent, un mode supplémentaire (à $680 \mathrm{~cm}^{-1}$ ) dont l'intensité est fortement dépendante du taux de substitution [5]. Ces deux techniques ont permis d'étudier un plus large corpus et de confirmer les substitutions systématiques mentionnées ci-dessus d'hétéro-atomes dans l'hématite et le corindon.

La figure 4 montre les résultats obtenus par spectroscopie Raman pour les principaux sites de production italiens et gaulois [6]. Les productions italiennes se démarquent clairement des productions gauloises. Il est même possible de distinguer les sites gaulois entre eux [5]. C'est plus difficile pour les sites italiens, pour lesquels la dispersion est plus importante. D’après les archéologues, les centres italiens comme Arezzo étaient en réalité un groupement d'ateliers plus ou moins indépendants, appartenant chacun à une grande propriété ou villa, et réalisant chaque étape du processus d'élaboration. Les centres gaulois et, notamment, celui de la Graufesenque, avaient une organisation plus industrielle, avec au moins des cuissons collectives. La préparation des argiles pour les pâtes et les couvertes pourrait aussi avoir été collective. Il est difficile d'établir des corrélations entre la composition chimique et les marques d'ateliers en Gaule, contrairement à l'Italie où il est possible de distinguer chimiquement les productions de certains ateliers d'un même centre.

\section{Valence du fer marqueur de la maitrise de la cuisson oxydante}

Nous avons vu plus haut que la présence de composés à base de fer divalent $\left(\mathrm{Fe}^{2+}\right)$ entraine un brunissement de la couleur de la couverte. Or, l'état de valence du fer dépend de l'atmosphère de cuisson, notamment lors de la vitrification de la couverte. Il est donc fondamental qu'elle soit suffisamment oxydante pour éviter la formation en trop grande quantité d'ions ferreux $\left(\mathrm{Fe}^{2+}\right)$ qui, une fois pris dans la matrice vitreuse, seront difficilement réoxydables. Le rapport $\mathrm{Fe}^{2+} / \mathrm{Fe}^{3+}$ est donc un bon marqueur de l'efficacité des protections des vases contre les fumées et gaz de combustion.

L'étude de l'état de valence du fer et de sa répartition dans les différentes composantes d'un vase (pâte, interface, couverte) a donné d'excellents résultats pour les céramiques attiques et campaniennes, avec la mise en évidence de procédés de cuisson complexes. Concernant les sigillées, le nombre d'échantillons analysés est encore trop faible pour en tirer des conclusions définitives [6]. Il ressort toutefois de ces études que les sigillées italiennes contiennent, en cœur de couverte, une proportion plus importante de $\mathrm{Fe}^{2+}$ que les

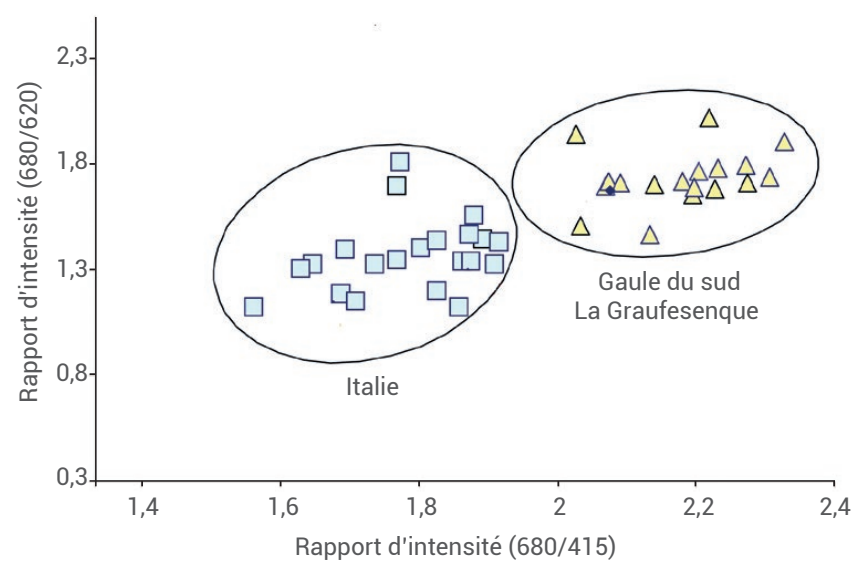

4. Séparation des deux régions de production (Italie et Gaule) sur la base du spectre Raman des cristaux d'hématite. La discrimination est basée sur les rapports d'intensité entre la raie supplémentaire $\left(a ̀\right.$ $\left.680 \mathrm{~cm}^{-1}\right)$ et les raies à 415 et $620 \mathrm{~cm}^{-1}$ [5]. 

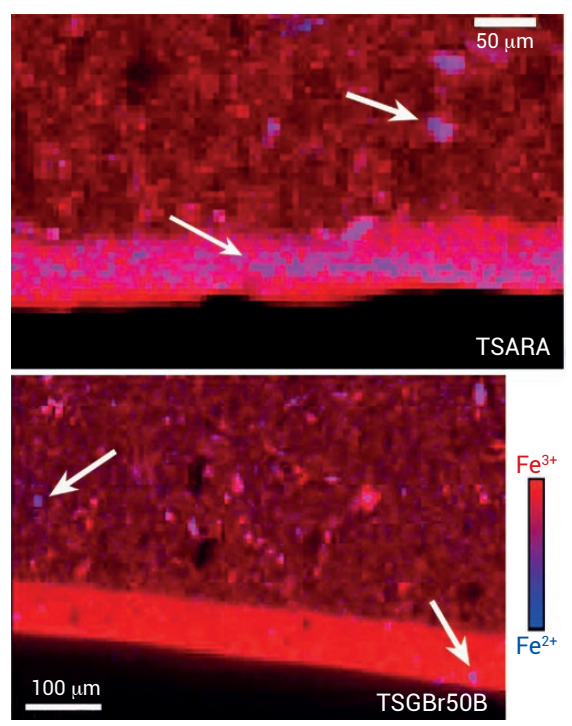

5. Cartographies de la répartition du rapport $\mathrm{Fe}^{3+} / \mathrm{Fe}^{2+}$ pour une sigillée italienne (TSARA) et une sigillée gauloise (TSGBr50B), obtenues au Stanford Synchrotron Radiation Lightsource (USA) par mesure de la fluorescence $X$ au seuil d'absorption $\mathrm{K}$ du fer [6]. Les zones riches en $\mathrm{Fe}^{2+}$ sont indiquées par les flèches.

$\gg>$

gauloises (fig. 5). Les couvertes des sigillées gauloises analysées ont été vitrifiées dans de meilleures conditions oxydantes que leurs homologues italiennes. Si cette situation se révélait générale, cela pourrait signifier que le système de canalisation des fumées et des gaz de combustion au moyen de tubulures, développé en Gaule du sud, était bien plus efficace que les solutions utilisées en Italie un demi-siècle auparavant. Les tubulures ne semblent avoir été introduites en Italie qu'au cours du $1^{\mathrm{er}}$ siècle après J.-C., soit à la toute fin de la période de production, et ceci peut-être grâce aux développements réalisés en Gaule.

\section{: Glossaire}

Terra sigillata Nom donné par les archéologues du $19^{\mathrm{e}}$ siècle en référence à I'utilisation de poinçons (ou sigilla en latin) pour la décoration des moules servant à obtenir les formes décorées.

Couverte

Enduit vitrifiable dont on recouvre les céramiques pour leur donner un aspect brillant.

Casettes

Étuis en terre réfractaire cuite, protégeant faïences et porcelaines de l'action directe du feu pendant la cuisson. (www.larousse.fr/ dictionnaires/francais/casette/13564\#ZAJD3WA8msSjYeSP.99)

Illites

Groupe de minéraux argileux dont la structure cristalline est constituée d'une alternance de trois couches : une couche d'oxyhydroxyde d'aluminium entourée de deux couches de silicates.
Il est rare que les matériaux du patrimoine se résument à des systèmes modèles. Ces matériaux se caractérisent non seulement par leur complexité, mais également par leur grande variabilité. Il s'agit là d'une difficulté incontournable : elle impose de bâtir une stratégie d'étude appropriée, qui consiste à conjuguer les apports de techniques permettant d'explorer des échelles spécifiques, sachant que les techniques offrant une haute résolution spatiale ne permettent de sonder qu'un petit nombre de volumes réduits. On se trouve confronté à des exigences antagonistes, entre résolution et représentativité. Dans ces conditions, chercher à dégager des conclusions ou lois générales de l'étude de ces systèmes complexes requiert de mettre en œuvre une approche multiéchelles et de maitriser l'échantillonnage. Dans le cas exposé, les analyses globales ont mis en évidence des caractéristiques propres à chaque grande zone de production. À l'inverse, les analyses locales, réalisées sur un petit nombre d'échantillons, ont permis d'en comprendre l'origine. Sans être en mesure de modéliser le système, l'approche multi-échelle permet de révéler les grandes tendances dominant les relations entre structure et propriétés physiques.

\begin{tabular}{|l|}
\hline Références \\
1• https://en.wikipedia.org/wiki/Terra_ \\
sigillata \\
2. Carte archéologique de la Gaule. \\
12. Aveyron (dir. Ph. Gruat, G. Malige \\
\& M. Vidal), Éditions de la Maison \\
des sciences de l'homme, Paris (2011). \\
3. Y. Leon et al., "Evolution of terra \\
sigillata technology from Italy to Gaul \\
through a multitechnique approach", \\
J. Anal. At. Spectrom. 30 (2015) 658-665. \\
4. Ph. Sciau et al., "Microstructural \\
and Microchemical Characterization \\
of Roman Period Terra Sigillate Slips from \\
Archeological Sites in Southern France", \\
J. Am. Ceram Soc. 89 (2006) 1053-1058. \\
5. Y. Leon et al., "Micro-Raman \\
investigation of terra sigillata slips: \\
a comparative study of central Italic \\
and southern Gaul productions", \\
J. Raman Spectrosc. 41 (2010) \\
1260-1265. \\
6. Ph. Sciau et al., "Reverse engineering \\
the ancient ceramic technology \\
based on X-ray fluorescence \\
spectromicroscopy", J. Anal. At. \\
Spectrom. 26 (2011) 969-976. \\
\end{tabular}

